\title{
Linguistics of Russian-speaking Kazakh musicology
}

\author{
Gulzhan S. Suleyeva - Gulzada M. Zhaxymbetova - Ainur Z. Mashimbayeva \\ - Raigul M. Meiramgaliyeva - Svetlana S. Janseitova
}

\section{DOI: 10.18355/XL.2017.10.04.02}

\begin{abstract}
The musical terminological system is analyzed in order to determine the linguistic status of the professional sublanguage of Russian-speaking musicology. The sources of the factual material were musicological and literary texts in Russian, containing translations of professional sublanguage musicology, dictionaries and works of Kazakh composers (nearly 3000 terms and 2000 terminological remarks). The complex description of musicological terms is given from the perspective of cognitive linguistics. There were revealed the tendencies to ethnographism in Russian-speaking musicology of the Kazakhstan. It is proved these tendencies are inherent to other countries in the region in varying degrees, which art is prevailed with the Russian. To the synthesis position of intercultural relations, characteristic of the expressive stylistic features of the composer's music remarks are provided. The nature and the features of sublanguage musicology are also defined. The results can be used to develop the technologies of mastering the characteristics of a codified vocabulary of professional Russian-speaking sublanguage musicology, its term system, model of the cognitive structure of the scientific text. Specially selected set of methods for the classification of the musical terminology remarks of analyzed musicology can be used in countries where the Russian language is used in many areas of the humanities.

Key words: linguistic cultural concept, sublanguage, terminological remarks, musical note, Kazakh musicology
\end{abstract}

\section{Introduction}

The terminology is a core component of the professional language of musicology (Monelle, 2014; Romero, 2016: 1; Beard and Gloag, 2016). Nevertheless, if the music art is in a state of rapid development and transformation, then the language elements, that serves it and the language itself undergoing qualitative changes. These processes are especially active and unpredictable in a situation when the professional Russianspeaking language musicology exists in some, not Russian, and not even the Slavic culture (Micklina, Konson, Zorin, 2016: 16).

Hence, the necessity of a complex analysis of the terminology with its term system, including the set of the terminological remarks, the need to study the relationship of composer's style and expressive speech means of the musical intonation.

Thereby, the questions of the explication of meanings and the context and style sense of the term and musical notes, the specificity of their expression, impact and perception of music by the performers and listeners also require the theoretical generalization.

In the scientific community has already drawn attention to the author's style features in the use of terms (Holoman, 2014; Kockaert, Steurs, 2015). The compositor's works of Haydn, Beethoven and Mozart, as the representatives of the classical system of music notes, have been studied to reveal their intention.

There is a problem of the origin of the musical notes science. According to Leychik (2007), the initial direction of the musical terminology was associated with a response to the inability to explain the phenomenon of lexical asymmetry of the term like a means of indicating a scientific concept (synonymy, ambiguity, formal variation) using the traditional methods. Therefore, analysing the term, we tried to transit from

XLinguae, Volume 10, Issue 4, October 2017, ISSN 1337-8384, eISSN 2453-711X 
the language system into a real verbal functioning of a term. Further in-depth study of the terms functioning had led us to the fact this problem has become one of the central in the terms studding and received a special name: the term and the text.

Perezman (1978) calls the remarks "musical terms", "the performing terms". She refers the "performing" and "scientific" musical terms to musical notes, later replaces them with the concept of "images". In the contrast to this theory, in the handing of Khayutin (2016) the remarks are not terms or metaphors, but are off-system lexemes: "terminological units, which are terms by their function, but in content, are not one of them". The remarks are also regarded as the imperative sentences with different lexical content.

We agree that music remarks have not only the performing function, but also the "composite" in the figurative structure of a musical work and consists of the scientific (the term), performing (the order), intonation and artistic (the image) content (PérezSobrino, Julich, 2014: 298; Antovic, 2009: 184).

Analyzing the literature on the topic of composer's professional remarks in their musical works it was found that this issue was given a little attention in the world of science. The researchers generally focused on the musical terminology, but did not consider the features and stylistics of cultural influence on the classical system of musical terminology.

In recent decades, the musicologist's interest to the creation of a musical style theory has been significantly greater than before. The larger circle of researchers refers to the consideration of the style in the performing interpretation (Krumhansl, 2015: 20; Nakamura, Takaki, 2015: 109; McKerrell, 2015: 614; Heero, 2007: 15). It forces us to explore the sublanguage musical elements even further.

The linguistic aspect is particularly important for our studding of the professional sublanguage Russian-speaking musicology. Many scientific sources analyse the general sublanguage characteristics. Most authors use the term "functional language" as a description language, and put it on a par with "the language for specific purposes", which is a means of communication in each of the specific spaces (Lekachman, 1999: 119; Lasswell, Leites, 2011; Mandell, 2011; Andrews, 2012). The researchers believe that the terminological expression, which is called professional sublanguage, is the most effective, so we use it in our work.

The content of each field of knowledge has its own sublanguage as a certain minimum set of lexical and grammatical categories and elements necessary for communication in a restricted field of activity and for description of a particular subject domain (Borgognone, 2003; Akhmanova, 2004; Solnyshkina, 2006).

According to the researchers, the introduction of the term "sublanguage" allows us to consider language as a complex of sublanguages (Pravikov, 2007; Zemlyanaya, Pavlycheva, 2010; Buyanova, 2015; Felde, 2015: 178). This term is arbitrary, but it is clearly defined and justified by the function features.

Thus, the opinions about the linguistically status of the professional sublanguage together with thought what methodological base we should use to study it - disagree. Available works have segmental and unsystematic feature, they are limited in describing the term groups, selected according to the principle of equal object reference, in studding of individual facts, related to the ontology and the functioning of the terminological vocabulary. In addition, the features of the existents of a professional sublanguage in a variant language environment have not considered at all.

In this regard, the aim of our research is the generally analysis of the musical terminology system in order to determine the linguistic status of the professional sublanguage of Russian-speaking musicology in Kazakhstan. To do this are being solved the following tasks:

- Description of the musicological terms from the perspective of the cognitive linguistics. 
- Identifying the trends of the ethnography in the Russian-speaking musicology in the Kazakhstan republic.

- Identifying and characterizing the expressive and stylistic features of the composer's music remarks from the position of synthesis and intercultural relations.

- Determination of the nature and features of the sublanguage musicology.

\section{Methodological Framework}

The carcass of the language units is about 5,000 units: 3000 terms and 2000 terminological remarks.

The sources of factual material were the musicological and the literary texts in Russian, containing translations of professional sublanguage of Russian-speaking musicology (al-Farabi, C. Valikhanov, A. V. Zataevich, A. K. Zhubanov, K. K. Zhubanov, M. Auezov, P. Aravin, B. Yerzakovich, K. Temirbekova, B. Sarybaeva, E. Tursunov, M. Margulan, M. Magauin; A. Asafiev, V. V. Medushevsky, E. Nazaikinskii; A. Muhambetova, B. Amanova, G. Omarova, A. Raimbergenova, S. Ayazbekova; S. Seifullin, S. Zhunusova, A. Tarazi); dictionaries, Kazakhstan composers works - the score and claviers (G. Zhubanova, E. Rakhmadiyev, A. Bestybaeva, A. Zataevich).

The research about the nominative nature of the phraseological combinations were carried out on the basis of the typology semantemes developed by Kopylenko and Popova (2010). This typology distinguishes five varieties of semantemes:

1) Denotative semantemes that reflects the extra linguistic nature directly: vocal music, symphonic orchestra.

2) Denotative semantemes designating a referent of a denotation, which is similarity or contiguity with other denotation: intonation fabric, minor logic. Compared with the first semanteme, the second includes new ones, not correlated with the composition of semanteme №1. This is a new semanteme created independently, but with the assistance of one or more semantemes.

3) Connotative motivated semanteme, which is not reflecting denotation directly, but is covered by the lexeme, which serves as a second (third) name of denotation. It carries a special evaluation function and the imagery, and is perceived only in conjunction of one lexeme with other lexemes. Connotative semanteme №1 is logically associated with denotative semantemes of the same lexeme, and is motivated by it: deep sound, reflecting sound. The main way of development of these semantemes is a metaphor. The metaphorical way to develop the semantemes due to the similarity of a particular quality of the correlated concepts.

4) Semantemes that do not have any motivated lexical connection with denotative semantemes of the same lexeme, for example: to cry as a white sturgeon, good riddance. This semanteme expresses the extreme rate of metaphor.

5) Isolated connotative semanteme, which does not have its own components and is completely unwarranted, isolated from other lexical system semantemes that has lost an active morphological form, for example: turn one's tail. It is the result of a very limited compatibility carrying by its lexeme: tweedledum and tweedledee.

Denotative and connotative semantemes, connecting in various combinations, form the phraseological combinations with two or three lexemes.

\section{Results and Discussions}

As specified compositional intent and verbally instructions, the terminology remarks appeared in notes in the eighteenth century and became an inherent part of musical notation (Losev, 1982). The high level of the performance skills and theoretical concepts of the Italian school had an impact on the development of European musical culture. This fact contributed to the spread of Italian terminological remarks in Kazakh composer's works. The carcass of remarks in the modern Kazakh 
compositor's works includes mostly Italian, Russian and Kazakh phraseological combinations and lexemes. This layering is caused historically (Vinogradov, 1963). The Italian terminological remarks are present as a foundation of this layer; Russian terminological remarks reflect the influence of Russian musical culture on the development of musical culture of European type in Kazakhstan. The Kazakh terminological remarks are a phenomenon that has social and aesthetic roots (focus on national artist), for which there is no equivalent expressions in other languages (Momynov, 1978). This was the cause of the appearance of non-Italian remarks in all musical cultures.

The Russian terminological remarks were a link between the original Italian and Kazakh remarks, performing the role of the guide thought the cultural contacts and were very important to familiarize the Russian people with the Kazakh music (Kopylenko, 1990: 1). The authors used the remarks recording and processing the sheets to convey the complex forms of Kazakh melody and the specifics of their performance (Kopylenko, 1990: 1).

The following results were based on the analysis of the actual material. The derivative terms of Russian-speaking musicology are formed in the following ways of word formation:

1) Morphological:

a) affixed (234 nouns, 87 verbs, 95 adjectives, 18 adverbs);

b) univerbation (108 nouns);

c) compounding (29 nouns $-2.2 \%)$.

2) Lexical-semantic (386 nouns, 53 verbs, 19 adjectives, adverbs 5).

3) Conversion (12 adjectives).

4) Drawing (125 nouns, 32 adjectives, 14 verbs, 66 adverbs).

Total number - 1283 units: 882 nouns, 154 verbs, 158 adjectives, 89 adverbs. There is a tendency to selectivity and regularity of the functions of word-formation models in the musicological terms. The selectivity is observed in the lexical and grammatical word classes' usage: dominated by nouns, adjectives and adverbs. The correlation of the parts of the speech in professional sublanguage musicology in favor of the noun indicates its nominative nature (Felde, 2015: 178).

Thus, we allocate two trends in the remark usage serving the Kazakh music:

1) In the professional genres - symphonies, sonatas, ballads, nocturnes, etudes, etc. is used the Italian remarks terminology, the example, of the works of M. Tulebaev, G.

A. Zhubanova, E. R. Rakhmadiyev, K. K. Kuzhamyarova.

2) In the records of Kazakh folklore, as well as in the works of professional composers for the folk instruments, ensembles and orchestras are used the Kazakh terminological remarks - semantic tracings of Italian original and Kazakh remarks.

All of these terms of musicology constitute a specific and isolated imagery language system that serves the music industry at the cognitive level, and which can be defined as a sublanguage.

Thus, the professional sublanguage of Russian-speaking musicology is a historically constituted and stable kind of a national language relative to this period, which occupies a subordinate position in the national language system. Sublanguage has the following characteristics:

1) Functionally limited in speech.

2) Serves the sphere of professional communication related to the concept system of the certain knowledge industry or field of activity (terminological space).

3) Has its own system of interacting sociolinguistic norms.

4) Happens to be a collection of the phonetic, syntactic, grammatical and preferably specific lexical resources serving certain verbal communication society; characterized by the union of the professional activities of its corporate societies and the corresponding system of specific concepts, a set of language elements and their relationships in the texts with limited subjects. 
5) Aspires to maximize the accurate expression of objective musicological thinking.

6) Reveals an intention to internationalization.

7) It is a universal means of describing the musicological facts and phenomena, characterized by the functional possibility of scientific knowledge progress associated with the changes in scientific concepts, theories and paradigms.

8) It is a unique way of describing the scientific facts and phenomena, characterised by the functional possibility of scientific knowledge progress associated with the changes in scientific concepts, theories and paradigms.

9) Does not have any analogs in non-musical world, but disposes a complex set of methods in order to analyze the audio material.

10) Is characterized by the tendency to involve into the composition the non-verbal means, which purpose in this field of language is quite diverse.

Linguistically professional sublanguage is also characterized by a certain idiomatic and stylistic limitations, providing mutual understanding between the scientists and the ability to recognise the results of their research. Mediating and optimizing the process of scientific communication, professional sublanguage contributes to the knowledge development and improves the information picture of the world. The sublanguage's main factor is to express the special concept accurately and in this manner facilitate the communication in the professional field of knowledge and professional activity.

The main features of the professional sublanguage are the systematization, the correlation with the particular space of applications, category features, written and oral forms of realization, non-verbal means usage (numeric, alphabetic, graphic). Thus, the professional sublanguage appears in its functional and structural orientation in the form of the phenomenon, which in concrete terms of the implementation separates into different professional species quite easily. As a language subsystem, the professional sublanguage retains its characteristics and, therefore, is seen as a means of generalization, accumulation and storage of information, serving the society, or rather, that part of it, which is associated with a certain professional use. As a means of scientific communication, it realizes the intellectual and the communicative functions, aiming for the objective, accurate, logically clear and purposeful transfer of the scientific information.

The professional sublanguage specific of the Russian-speaking musicology (PSSRSM) is the emotional and sensual musical comprehension of reality, hence, the emotional regulation of man's relationship with the world of sounds. Musicology, like any other scientific discipline, needs a sublanguage, since in the course of its evolution the accumulated scientific knowledge reached a certain stage of maturity and has been looking for a sign system, language of expression. The forms of the scientific knowledge sign illustration differ: by code, the language for writing knowledge contents in a form of symbols, words, phrases, sentences; different types of texts, where the scientific knowledge finds its clearance; logical-conceptual knowledge system; terminological system.

The core vocabulary of the PSSRSM is terminology, which is a relatively closed system, the composition of which is determined by the communicational area of musicians, composers, musicologists, and others, who uses this sublanguage. As the musicology foundation, without which it is impossible to master the basic provisions, its terminological system appeared as a special form of response to cognitive processes and professional activities. Most musical terms were introduced into practice by direct reference to the musical sound events, or by the artistic description.

As with any professional terminological system, the codified terminological units make a large part of the musicological terminology. With the highest level of the information content, they create its core and express the specific content of this field of knowledge: the sound, rhythm, meter, beat, scale, third, polyphony, harmony,

XLinguae, Volume 10, Issue 4, October 2017, ISSN 1337-8384, eISSN 2453-711X 
chord, instrumentation. However, some terms do not have the full properties such as accuracy and definitive. This is because there is often a need to express the difficult concepts related to the area of feelings, emotions, original insight: invading cadence, Melos blossoming, the top source, the quiet culmination, the gold path, wolf interval, seventh air. The semantic of the term structure is determined by two factors: the nature of the terminology and the linguistic substratum. The term receives its semantic structure from the language substrate. It includes the content (its own meaning), and the internal shape (sense, motivation, the way in which the content is presented, the meaning of a lexical unit and etc.).

The terminological Kazakh language system is a special linguistic, cultural and communicative phenomenon. The performing style of the Kazakh professional musicians, in contradistinction to European depends on the musician's current mood and is never occur again. This observation determines the complexity of the study of musical terminology in Kazakhstan.

The originality of the traditional Kazakh music is that it functions on the basis of nomadic life, did not have a city form of concert life or writing theory and the performance was combined with the socially significant forms of communication (the ceremonial, celebration, ritual, hospitality, inter-clan competition) (Tleubergenov et al., 2016: 5250). The Kazakh music carriers personified in the syncretic forms the principle of universal totality; in Kazakh culture musician became widely known only if he had his own works, marked the print of a bright personality. The Kazakhstan musicologists, based on the Russian and international language, have created the terms, which reflect the specifics of the Kazakh people's musical thinking, they have successfully implemented the flexible natural transition from the traditional to professional terms.

In translating the content into form of expression, which is realized in the process of generating the symbol, sound forms and musical events, the musical form are abstracted in the form of the language elements - the terminological remarks. The terminology remarks - a sound recording system, transmission the musical fabric structure, acoustic, rhythm, tempo, instrument features, aimed to convey the composer's intent. The musical remarks in the note - words, phrases and graphic symbols, which are the settings for the musician and determine the essential features of the intonation and artistic images. We consider terminological remarks to be a reflection of the expressive speech aspects in musical intonation.

The composer's music language - a set of stable types of sound combinations (intonation) and the rules of their usage. Considering the content of this description to be an origin, we believe that the language of music and style is a set of intonation and artistic symbols, the rules of their organisation and typological musical and composite structures (or, as they say in musicology, musical forms).

In the sounding composition, the musical elements sound qualitatively and expressive by means of performing musical accent, which is prescribed by the composer. The modality concept is important, as it means the ratio speaking to the reality, speech content, and form of speech, emotional and expressive evaluation of the report. The set of modalities, their selection and organisation can serve as indicators of the style of composer's speech.

Remarks are off-system lexeme, as they function as transition from the significance, mythological or artistic world images (inside the arts) to the music and art, and then to the scientific, to the conceptual system of reflection attributes, enclosed in them.

Among the functions of musical remarks, there are a motive, expressive, emotional, communicative and figurative associative functions. Musical remarks are determine the nature, individualization of performing with a certain proportion of relativity, contributing to the understanding of the style, content, mood and poetic nature of music.

Let us consider the musical terminological remark espressivo 'expressive, 
emphatically', which often follows the remarks, related to categorical fields of communication and experience. In the musical and verbal sense the remark espressivo means - with particular power and increased energy of inner experience, its personal sense of realisation in intonation and artistic images. Remark espressivo enhances the remark "very gently, joyfully and easily", thus ensuring the preservation of the softness performance. The expression may be contrasting, expressive, quiet expressive, mid-quiet expressive, wide expressive.

In the analysis of the musical remarks and their groupings, was used the stylisticalstatistics method with the following systematization. The remarks based on common features have been combined into the lexical, conceptual and categorical fields. Lexical field, included the family of words formed by any one certain word, is united around a specific remarks. For example, remarks allegro 'fast', allegro assai, allegro con moto, allegrissimo 'as fast as possible' are included into the lexical field allegro 'fast'. The conceptual field includes the system of interrelated concepts, organized around a central concept. Herewith, the degree of the central concepts generality may be different. For example, you can combine the lexical fields allegro, vivace 'lively', presto 'fast' into the conceptual field of fast tempo 'time'. You can merger the term groups of slow, moderate and fast into one conceptual field - tempo. The tempo together with the characteristics of human motion and nature (flightiness, stumbling, rotating) will be included in the categorical field movement, which is one of the most common categories of being, and in the context of intonation and artistic image of the world will mean the musical movement.

The remarks contain a huge number of concepts with varying degrees of generality and specificity, for example: the impulsive passion and intense pathos, spontaneous emotions - longing, anxious or flaming combustion. Especially diverse is the usage of poetry modalities by the composers, which have no analogues in the conventional remarks, for example: the rhythmic pattern in sad and cold landscape coloring, subtle dynamic thrift. Choosing a language means in order to implement the intention of speech, the author projects on the text the personality traits that in interpersonal communication is interpreted by the recipient and are a signal that regulates their subsequent verbal behavior.

The recognition of the idea of folk theory existence as priceless cultural and spiritual achievements of the Kazakh people allowed considering it as a cultural realities in the contemporary musicology, as the source and basis of the PSRSKM formation. The analysis of the ethnic and cultural traditions system, combined into a single entity in the context of diverse philosophical, conceptual and artistic generalizations, conceptual-categorical norms and the representations of the Kazakh musical culture, allowed to understand the concept of traditional music in terms of reflecting the people's mentality, the features of their world perception.

Classifying the musical remarks, we proposed an original principle of their categorization by the structure image of the world: spaciousness, objectivity, movement, temperament, feel, experience, intellection, will, action, behavior and communication. In the subsequent generalisations, the quantitative and qualitative remark's analysis allows the transit to a non-trivial stylistic concepts reflecting expressive speech style, such as soft style, witty, sad, plastic, willful and emotionalmotor.

For the first time, the expressive music speech style is considered as an independent historical composing and performing category, living and evolving, not only in close unity with the musical-linguistic means, but also independently of them. The theory and the musical articulations classification, its interpretation technique in the stylistic aspect are a principle new classification.

In the Kazakh musical terms the creative thinking is expressed not as a development and procedure, but as a state of soul. A feature of the traditional musical concepts is

XLinguae, Volume 10, Issue 4, October 2017, ISSN 1337-8384, eISSN 2453-711X 
the ability to transmit the attitudes toward the world as an expression of the unspeakable - the immersion into emotional condition, meditative, detachment from the real world, from the empirical reality. In the existence of such genres as kui 'traditional Kazakh, Kirghiz instrumental play', akzhelen 'short gay traditional Kazakh, Kirghiz instrumental play - short form of kui', were crystallized the musical concepts that reflect the processes of shaping the musical rhythm, musical intonations that have been passed down orally from generation to generation. The traditional musical term's musical-esthetic and communicative functions combined with the phenomenon of the traditional culture in the organic unity.

Studying the cultural-laden language units, we proceeded from the assumption that language - is an essential component of the people's perception formation, which is reflecting the ethnic history, its material and spiritual culture, which expresses, in addition to the language content, the cultural knowledge. The processes of creation, oral musical work transmission, fixation it in the universal cultural memory were possible thanks to the preserved linguistic-cultural concepts, by which were expressed the music concepts and presentations, developed in the Kazakh language over the centuries. If Russian vocabulary is based on the West European and Russian terms, the Kazakh - on the West European, Russian and Kazakh terms. The traditional Kazakh music was consisted of two main layers - the folklore and professional music of oral tradition, the intermediate position between which took the amateur musicmaking and the non-rite music layer.

The particular interest is caused by the changes in the ethnic cultural semantic lexeme $k u i$, having not only artistic and aesthetic musical genre form, but also expressing the Kazakh's perception of the world. The base meaning of kui (state) fixes one of the essential manifestations of the world in a person's sensations. Reflecting of the different conditions in the instrumental music, which is the encyclopedia of the imaginable-emotional people's life, transformed the cultural meaning of kui (the piece for musical instrument). The connection of the musical term kui with the ideological category of condition reflects the essence of the ancient Turks music, composed on the feeling of being as a stream of divine states, the pure conductor of which is music. A century of the people's musical performances experience is compressed in the traditional musical concepts. They are characterized by empirical concreteness, ambiguity, comparable with similar phenomena and apparent objective environment (bass byun 'uppercase, first register', Saga 'saga').

The Russian-speaking Kazakh literary texts analysis has shown that they are widely represented with the non-equivalent vocabulary, describing the specifics of the musical culture of the Kazakh people, rich instrumentation, a variety of genres and artists. Introducing the Kazakh origin words, which do not have regular reproducible Russian equivalents, into the fabric of Russian texts, the authors make from the Kazakh music a heritage of the realities of Russian language, therefore, the entire multi-national culture of Kazakhstan.

The meaning of the non-equivalent words, as well as any other word, consists of the minimal semantic components - semantemes, which are divided into nuclear and peripherals. Nuclear semanteme most fully express the generic term, when the peripheral - more concrete, specific concepts. The more semantic features lies in the meaning of words, the farther it is removed from the core. The main information on the entity field is focused in the core (as in the core of the concept). Classification of the denotative and connotative semantemes allows differentially describing the various cases of the semantic implementation of the non-equivalent words. The denotative semanteme, its component reflects a certain sign of an object or concept. There are ten denotative semantemes, which contrast in distinctive features. We confine ourselves to the three denotative semantemes:

- in their relation to the language system: common usage (are included in the system word meaning) and occasional (are imposed by the context or situation, aren't 
included in word meaning, but joins it in the communicative act);

- by their distinctive power: integrated (common for the word meaning groups) and differential (operating different functions). The archesemantemes are a variety of the integrated semanteme, pertinent the subject to a certain class. Identifying the archesemantemes is caused by the necessity of an object categorization;

- by the specific content nature: assertiveness and dispositional.

Kazakh words in Russian texts without the semanteme updating are able to provide the necessary installation on the local coloring creation. The actualisation only complements the national characteristic of the indication of particular named object usage. The semanteme actualisation is a communicative due to the allocation of the semanteme in the meaning's structure, leading to the perception of its participants the act of communication as the communicative-relevant, which is re-entrant of the actual word meaning.

The non-equivalent words we systematised the theme feature. With regard to musical terminological system are discern the following: the name of song and instrumental genres; names of the musical instruments; names of the Kazakh music performers.

By updating the differential semantemes the authors define the attributes of Kazakh folk musical instruments, reflect their national identity, reported an acoustic and musical-technical features of the instrument, its formation, sound scale, dynamic shades, richness of performing traditions; distinguish the attributes of Kazakh life as an important component of the national culture, with their cultural meanings and its place in this world.

The non-equivalent words are charged with varied connotations. The words, which reflect the national identity, national soleness, cannot be replaced by the words belonging to the native (in this case Russian) lexicon. The interesting dispositional semanteme lexeme is kobyz. 'Kazakh national bowed stringed musical instrument'. Semantemes such like these are updated in the artistic and musicological texts more often than others:

a) "instrument magical function", "shaman's instrument", "the intermediary between the shaman and the spirits", "the fate overlord". For example:

In the shaman's hands, during the religious acts, kobyz was the mediator between the people and the invisible, powerful spirit world, surrounding the people and affect their lives (Raimbergenov, 1990);

b) "anthropological structure of the instrument design", "connection with the cosmic harmony". As an example:

Thus, the belly and two bridges placed on it and regulating two strings, represent the Upper World. The hollowed part of the kobyz as the two brain halves, represent the Mid-World. The underworld - the kobyz bottom is nothing else as the waters Chaos. The touching of the bow and two strings as the two forms of energy - solar and lunar, male and female ... (Raimbergenov, 1990);

"In the shaman's activities the kobyz was a tool, which helped him enter the trance" (Raimbergenov, 1990); "Hidden and unexplained energy and power of an instrument", "instrument magical function, with which you can travel in time and space", "instrument aesculapian function";

c) "Prediction", "foresight", "zhyrau instrument". For example:

It is no coincidence that the zhyrau was taken in military campaigns, as kobyz could predict the outcome of the forthcoming battle (Raimbergenov, 1990).

Daulpaz 'huge drums' are the signal, hunting and military instruments. They have caldron-looked wooden shell, the hollow part of which is tight with the cowhide. The

XLinguae, Volume 10, Issue 4, October 2017, ISSN 1337-8384, eISSN 2453-711X 
bow is arc-shaped, which is stretched with two strings of non-woven horsehair, and the metal pendants are hung on the top. The texts are updated with such dispositional semanteme lexemes of daulpaz: "awesome sound range of the instrument", "an instrument of power dictates", "an instrument of will suppress"; "a signal", "battle cry", "the submission", "the call to act, to fight, to conquer", "power of sound", "fear", "power of deterrence", "execution". The inclusion of non-equivalent words in the artistic and musicological texts considerably enriches the emotional palette of Russian texts with the number of unique, unusual for the Russian reader semantic overtones, introducing it into the circle of bright new ethnographic realities.

The performing means of musical expression also can be composer's, as they starting from a certain historical time, in most cases, expressed their requirements of performing in remarks (verbal or graphic), and accordingly - of the nature of figurative content of sounding music. The researchers faced with the task to make understand in a unified concept the traditional and new notions, related to the means of performing the pronunciation. There are many disagreements in the concept's interpretations, relating to musical and verbal means of expression, and therefore, musical technique requires a theory that could provide guidance for quite a reasonable interpretation of the meanings and context style senses, contained in the musical remarks.

As a rule, the professional sublanguage suggests the communication between the members of a certain professional group, who are solving and discussing the professional tasks, problems, decision making between the professionals on a professional theme.

There are various points of view about the professional sublanguage status. Professional sublanguage is a special kind of a functional national language, serving the sphere of professional communication (Danilenko \& Skvortsov, 1981: 7). It is a special functional form of national language, serving the space of professional language (Avdeeva, 2013: 3). Some researchers also believe that professional sublanguage is a completely self-contained form of language existence (Korovushkin, 2005).

We define professional sublanguage as a functional kind of national language, the social limited kind, its smaller subsystem, which occupies a subordinate position and regards to it as part of the whole.

The international feature of the Russian-speaking professional sublanguage musicology, for our definition, is caused, on the one hand, with the external factors, such as community and the interpenetration of musical achievements in the world, on the other hand, with the inter-institutional abilities of the Italian language, which allowed developing a lot of branches of the musicology's terminology. The most numerous group of the musicological terms are borrowed from the Italian language, which is the most widely used in the music space and is ideal to transmit all the shades and subtleties of thoughts and feelings in music. The structure of borrowed terminology vocabulary includes the terms, structurally identical to the foreign language prototypes, without any modifications to the structure; terms, morphologically designed by means of borrowed language; terms, with the partial morphological substitution, representing a hybrid vocabulary; terms, which are formed based on the semantic tracing.

\section{Conclusions}

Considering the existing in the musical culture traditional system of musical terms, we came to the conclusion that they are fundamental for the systematization musical material, creation of the folk theory, which reflects the essential features of ancient musical culture. As the phenomenon of language, culture and consciousness, the folk theory is a carrier of the people's artistic energy and serves as one of the nationalspecific means of development of the world. The traditional Kazakh musical terms, 
which carry an ideological and sacral information, reflect the structure and the process of formation of the kui. Thanks to them, the equipment and techniques of playing the dombra, kobyz, the richness of the performance timbre, form of kui have been preserved for the future.

The modality of a musical work is found in the intonation, indicated by the composer in musical remarks, as a reflection of the essential features of the composer's expressive speech style. Terminology remarks contribute to the deployment of thematic text musical scores, its pragmatic interpretation, and the development of the cognitive processes. The analysis of terminological remarks revealed different levels of artistic structure - longitude, dynamic, agogical, timbre characteristics with its specific, historical, stylistic features.

An analysis of lexical, conceptual and categorical fields of music remarks in the music scores of Kazakhstan composers showed that the composer's expressive speech style is defined by its complexes of image attributes (motion, space, experience, sonority and behavior), expressed in the remarks and embodied in appropriate ways of musical accent. Dynamics, the sound and intonation pattern of the composition have their own specific, historical, imaginative features and stylistic shades that operate with the strictly regulated parameters of expression means. The complex is unique, inimitable in characteristic of the composer's expressive speech style, transmitting its particular melody, harmony and instrumentation.

The pragmatic analysis of the musicological texts (in particular, the music scores and claviers) allowed us to determine the features of the functioning musicological discourse by describing the basic strategies and tactics of composer's and performer's speech behavior.

As a symbolic expression of musicological concepts, a professional sublanguage of Russian-speaking musicology is the mass of scientific knowledge, which ensure the mutual understanding between the professionals in the music space. The main functions of the Russian-speaking professional sublanguage musicology are epistemic, cognitive and communicative, regulatory. Such features characterize it: systematic, correlation with a particular area of knowledge, regardless of the context, brief and clear-cut, internationally, nomination features, language realization form, non-verbal means and word-forming agent.

The professional sublanguage of Russian-speaking musicology has strong potential of means of formation terms, which ensure the optimal accessibility of professional musician's activity.

The analyzed traditional musical terms confirm the position that the traits of reality, which are essential, important for native speakers and culture find their expression in each language. The position that, mastering the language and culture, the native speaker begins to see the world from the standpoint of, prompted by his language, and combines with the conceptualization of the world, inherent in its culture.

The results of the research are aimed at solving the problems of representation of terminology, semantic, conceptual and functional aspects of scientific professional knowledge. So they can be used to develop the technologies of understanding the structural and semantic functional characteristics of a codified vocabulary of professional sublanguage of Russian-speaking musicology, its terminological system and model of a cognitive structure of scientific text.

\section{Bibliographic references}

AKHMANOVA, O. 2004. Dictionary of Linguistic Terms. Moscow: Editorial URSS. ANDREWS, E. 2012. A history of Scientific English: The Story of Its Evolution, Based on a Study of Biomedical Terminology. Whitefish: Literary Licensing, LLC.

ANTOVIC, M. 2009. Musical metaphors in Serbian and Romani children: An empirical study. Metaphor and symbol, vol. 24, n. 3, pp. 184-202.

XLinguae, Volume 10, Issue 4, October 2017, ISSN 1337-8384, eISSN 2453-711X 
AVDEEVA, V. V. 2013. Functional Varieties of Language: Linguistic Comparison of LSP and Sublanguage. Scientific notes of the National University "Ostroh Academy", vol. 35, pp. 3-5.

BEARD, D. - GLOAG, K. 2016. Musicology: the key concepts. Routledge.

BORGOGNONE, P. 2003. Problems of English Economic Terminology. Torino: Universitaria Levrotto \& Bella.

BUYANOVA, L. 2015. Terminological Derivation in the Language of Science: Cognitive, Semiotic Functional Aspects. Moscow: Flinta.

DANILENKO, V. - SKVORTSOV, L. 1981. Linguistic Problems Ordering Scientific and Technical Terminology. Journal of Questions of linguistics, vol. 1, pp. 7-16.

FELDE, O. 2015. Professional Sublanguages and Terminology of the Russian Language as the Object of Scientific Study. Tomsk State Pedagogical University Journal, vol. 4, pp. 178-184.

ZEMLYANAYA, T., PAVLYCHEVA, O. 2010. The Terms and Terminological Combinations. Main Characteristics of the Scientific and Educational Information Magazine, vol. 2, p. 40.

HEERO, A. 2007. Poetry and music. On the intermediality in the early writings of Robert Schumann. Trames-Journal of the Humanities and Social Sciences, vol. 11, n. 1, pp. 15-34.

HOLOMAN, D. K. 2014. Writing about Music: A Style Sheet. Okland: University of California Press.

KHAYUTIN, A. 2016. Term, Terminology, Nomenclature. Samarkand: Samarkand State University.

KOCKAERT, H. J. - STEURS, F. 2015. Handbook of Terminology. Amsterdam: John Benjamins.

KOPYLENKO, M. 1990. Types and Functions of Foreign Language Component in Artistic Texts. Kazakh word in Russian art texts. Alma-Ata, pp. 1-32.

KOPYLENKO, M. - POPOVA, Z. 2010. Essays on the General Phraseology. Moscow: Librocom.

KOROVUSHKIN, V. 2005. Contrastive Sociology as an Autonomous Linguistic Discipline. Cherepovets: Cherepovets State University.

KRUMHANSL, C. 2015. Statistics, Structure, and Style in Music. Music Perception: An Interdisciplinary Journal, vol. 33, pp. 20-31.

LASSWELL, H. - LEITES, N. 2011. Language of Politics: Studies in Quantitative Semantics. Retrieved from https://www.amazon.com/Language-Politics-StudiesQuantitative-Semantics/dp/1258135906

LEKACHMAN, R. 1999. The Language of Economics. In: Journal of Language in America, pp. 119-131.

LEYCHIK, V. 2007. Terminology: Subject, Methods, Structure. Moscow: Editorial URSS.

LOSEV, A. 1982. Sign, Symbol, Myth Works on Linguistics. Moscow: Moscow University Press.

MANDELL, A. 2011. The Language of Science. NSTA, 1742 Connecticut Avenue, N.W., Washington, DC.

MCKERRELL, S. 2015. Social distance and the multimodal construction of the other in sectarian song. Social Semiotics, vol. 25, n. 5, pp. 614-632.

MICKLINA, N. - KONSON, G. - ZORIN, A. 2016. Sociology of Music in Russia in the context of Globalization and National Identity. Global Media Journal, vol. 3, p. 16.

MOMYNOV, P. 1978. A Short Course of Musical Literature of Kazakhstan. AlmaAta: Zhalyn.

MONELLE, R. 2014. Linguistics and semiotics in music. Routledge, vol 5 
NAKAMURA, E. - TAKAKI, S. 2015. Characteristics of Polyphonic Music Style and Markov Model of Pitch-Class Intervals. Mathematics and computation in music. Proceedings of $5^{\text {th }}$ International Conference. London, pp. 109-114.

PÉREZ-SOBRINO, P. - JULICH, N. 2014. Let's talk music: A corpus-based account of musical motion. Metaphor and Symbol, vol. 29, n. 4, pp. 298-315.

PEREZMAN, L. 1978. The Formation and Development of German Musical Terminology and Its Expressive Stylistic Functions. Taskent: Taskent University.

PRAVIKOV, Y. 2007. Phenomenon in Sublanguage Culture in the Context of National Concepts, Literary and Artificial Language. Analysis of Cultural Studies, vol. 7.

RAIMBERGENOV, A. 1990. Kui Қaynary. Almaty: Oner.

ROMERO, B. M. 2016. Musical Semiotics as a Tool for the Social Study of Music. By Óscar Hernández Salgar. In: Ethnomusicology Translations, n. 2, pp. 1-33.

SOLNYSHKINA, M. 2006. Professional Marine Sublanguage. Moscow: Academia. TLEUBERGENOV, A. A. - JUMANIYAZOVA, R. K. - BEGEMBETOVA, G. Z. NUSSUPOVA, A. S. - KAIRBEKOVA, A. G. - KESHUBAYEVA, D. Y. 2016. Typological Features of the Kazakh Ethnic Picture of the World. In: International Journal of Environmental and Science Education, vol. 11, n. 12, pp. 5250-5260.

VINOGRADOV, V. 1963. Stylistics. The Theory of Poetic Speech. Poetics. Moscow: AS SU.

Words: 6890

Characters: $47170(22,8$ standard pages $)$

Associate professor Gulzhan S. Suleyeva, Kandidate of Philology

Gulzada M. Zhaxymbetova

Associate professor Ainur Z. Mashimbayeva, Kandidate of Philology

Associate professor Raigul M. Meiramgaliyeva, Kandidate of Philology

Professor Raigul M. Meiramgaliyeva, doctor of Philology

Department of Chair of social and humanitarian disciplines KNC

KNC under Kurmangazy

86 Abylai Khan Ave

50000, Almaty

The Republic of Kazakhstan

dzhanseitova.sv@gmail.com 